\title{
Risk factors for mortality in elderly haemodialysis patients: a systematic review and meta-analysis
}

\author{
Yu-Huan Song ${ }^{1,2}$, Guang-Yan Cai ${ }^{2^{*}}$, Yue-Fei Xiao ${ }^{1 *}$ and Xiang-Mei Chen ${ }^{2}$
}

\begin{abstract}
Background: Older haemodialysis patients accompany a high burden of functional impairment, limited life expectancy, and healthcare utilization. This meta-analysis aimed to evaluate how various risk factors influenced the prognosis of haemodialysis patients in late life, which might contribute to decision making by patients and care providers.
\end{abstract}

Methods: PubMed, Embase, and Cochrane Central were searched systematically for studies evaluating the risk factors for mortality in elderly haemodialysis patients. Twenty-eight studies were included in the present systematic review. The factors included age, cardiovascular disease, diabetes mellitus, type of vascular access, dialysis initiation time, nutritional status and geriatric impairments. Geriatric impairments included frailty, cognitive or functional impairment and falls. Relative risks with 95\% confidence intervals were derived.

Results: Functional impairment $(\mathrm{OR}=1.45,95 \% \mathrm{Cl}: 1.20-1.75)$, cognitive impairment (OR=1.46, 95\% Cl: 1.32-1.62) and falls $(\mathrm{OR}=1.14,95 \% \mathrm{Cl}: 1.06-1.23)$ were significantly and independently associated with increased mortality in elderly haemodialysis patients. Low body mass index conferred a mortality risk (OR $=1.43,95 \% \mathrm{Cl}: 1.31-1.56)$ paralleling that of frailty as a marker of early death. The results also confirmed that the older $(\mathrm{OR}=1.43,95 \% \mathrm{Cl}$ : 1.22-1.68) and sicker (in terms of Charlson comorbidity index) ( $\mathrm{OR}=1.41,95 \% \mathrm{Cl}$ : 1.35-1.50) elderly haemodialysis patients were, the more likely they were to die. In addition, increased mortality was associated with early-start dialysis $(\mathrm{OR}=1.18,95 \% \mathrm{Cl}: 1.01-1.37)$ and with the use of a central venous catheter ( $\mathrm{OR}=1.53,95 \% \mathrm{Cl}: 1.44-1.62)$.

Conclusions: Multiple factors influence the risk of mortality in elderly patients undergoing haemodialysis. Geriatric impairment is related to poor outcome. Functional/cognitive impairment and falls in elderly dialysis patients are strongly and independently associated with mortality.

Keywords: Dialysis, Mortality, Risk factor, Elderly, Geriatric, Aged

\footnotetext{
* Correspondence: caiguangyan@sina.com; 13910587886@163.com

${ }^{2}$ Department of Nephrology, Chinese PLA Generl Hospital, Chinese PLA

Institute of Nephrology, State Key Laboratory of Kidney Diseases, National

Clinical Research Center for Kidney Diseases, 28 Fuxing Road, Beijing 100853,

China

${ }^{1}$ Department of Nephrology, Aerospace Center Hospital, 15 Yuquan Road,

Beijing 100049, China
}

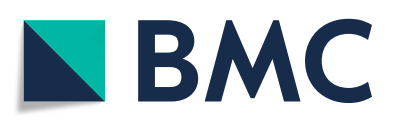

(c) The Author(s). 2020 Open Access This article is licensed under a Creative Commons Attribution 4.0 International License, which permits use, sharing, adaptation, distribution and reproduction in any medium or format, as long as you give appropriate credit to the original author(s) and the source, provide a link to the Creative Commons licence, and indicate if changes were made. The images or other third party material in this article are included in the article's Creative Commons. licence, unless indicated otherwise in a credit line to the material. If material is not included in the article's Creative Commons licence and your intended use is not permitted by statutory regulation or exceeds the permitted use, you will need to obtain permission directly from the copyright holder. To view a copy of this licence, visit http://creativecommons.org/licenses/by/4.0/ The Creative Commons Public Domain Dedication waiver (http://creativecommons.org/publicdomain/zero/1.0/) applies to the data made available in this article, unless otherwise stated in a credit line to the data. 


\section{Background}

Elderly end-stage renal disease patients constitute an increasing fraction of patients on renal replacement therapy worldwide [1]. The mortality rate of elderly dialysis patients remains confusingly high in spite of recent technical advances, especially in those with a high rate of multimorbidity, muscular functional impairment, cognitive defects or falls [2-5]. However, there is no consensus about the factors affecting the mortality of elderly haemodialysis patients [6]. In particular, survival is no longer the focus of care. The goal is to either improve the overall quality of life or at least meet some functional or emotional goals, which often entails successful living rather than mere survival. It is important for the need of estimating "geriatric syndromes," such as frailty and falls, to the risk-stratification of older dialysis patients and guiding treatment decisions [7].

This systematic review made an effort to achieve a broad-scale research of the accessible studies to recognize the risk factors for mortality in elderly haemodialysis patients. The goal was to evaluate the association of functional impairment, cognitive disfunction and falls with mortality in elderly haemodialysis patients, in addition to other known risk factors. Recognizing these associations might help improve treatment solutions or protective measures.

\section{Methods}

The study was written according to the Preferred Reporting Items for Systematic reviews and MetaAnalyses (PRISMA) guidelines and was displayed in keeping with the PRISMA-P checklist (Additional file 1).

\section{Protocol and registration}

No registered protocol.

\section{Identification of eligible studies}

A systematic literature search was carried out using the PubMed and Web of Science databases from inception to November 9, 2019. The following terms were used to perform the search: 'dialysis', 'dialys"', 'renal dialysis', 'interdialysis', 'inter-dialy*', 'hemodialysis', 'hemodialys*', 'haemodialysis', 'haemodialys*', 'aged', 'elderly', 'geriatric', 'mortality', 'survival', 'risk factor', 'functional impairment', 'cognitive impairment' and 'falls'. The detailed search strategy is shown in Additional file 2 (Additional file 2). Only studies in English were accepted.

\section{Data extraction and risk-of-Bias assessment}

Two authors, YHS and GYC, independently displayed the list of studies generated by the search, with disagreements resolved by a third author, YFX. Titles and abstracts of all studies were screened before acquiring full-text versions of relevant studies. Two authors extracted data from fulltext articles independently.

Inclusion criteria: (1) risk factors for mortality of elderly haemodialysis patients were the subject; (2) haemodialysis patients included an elderly population; and (3) study data included odds ratio(OR) values and 95\% confidence intervals (CIs) or data that could be transformed to OR values and $95 \%$ CIs by statistical methods.

Exclusion criteria: (1) the abstract was not in English; (2) the study did not involve elderly haemodialysis patients; and (3) it was a case report, abstract, review, conference report or animal experiment.

The quality of articles was accessed using the Newcastle-Ottawa quality assessment scale [8]. Studies below 5 points were accounted to have a high risk of bias and were excluded.

\section{Data collection and analysis}

The data included authors, year of publication, number and mean age of participants, percentage of men, median duration of follow-up, survival or mortality, risk factors, and definition of ageing. The risk factors mainly included age, cardiovascular disease, diabetes mellitus, type of vascular access, dialysis initiation time, nutritional status and geriatric impairments. Geriatric impairments included frailty, cognitive or functional impairment and falls. Relative risks with 95\% CIs were derived. We extracted the adjusted hazard ratios (HRs) and 95\% CIs from all included studies.

\section{Statistical analysis}

We evaluated the pooled relative risk and the $95 \% \mathrm{CI}$ of the included articles through the inverse variance method. ORs of retrospective studies were considered as approximate risk ratios (RRs). We used the $I^{2}$ statistic and $Q$ test to account the heterogeneity among the included studies. No significant heterogeneity was present if the $I^{2}$ statistic value was $<50 \%$. Then we used a fixedeffect model to calculate the pooled 95\% CI. If significant heterogeneity was showed $\left(I^{2}\right.$ statistic value was $\geq 50 \%$ ), we used the random-effect model. Review Manager 5.3 was used for statistical analyses.

\section{Publication Bias}

We evaluated Publication bias by Egger's and Begg's tests at the 5\% significance level. Point prevalence with 95\% CIs was showed in the forest plot pattern. A funnel plot was used to evaluate the publication bias.

\section{Sensitivity analysis}

We accessed the OR value and 95\% CI of each risk factor under the fixed-effect model and the random-effect model separately. If the difference between the two results was small, the combined results had low sensitivity and stability. 


\section{Results}

\section{PRISMA flow chart}

The overall literature search generated 6785 articles. Of these, 291 articles were selected on the basis of the inclusion and exclusion criterion of the literature. We excluded 211 irrelevant studies after reading their titles and abstracts. Thus, 83 latent full-text articles were appraised for qualification, which brought about further exclusion of 55 articles because the result of interest was not demonstrated. After all, 28 articles were in line with the suitability criteria and were included in this metaanalysis. The flow diagram of the study screening procedure was showed in Fig. 1. The features of the 28 articles were summarized in Table 1.

\section{Risk factors for mortality in elderly haemodialysis patients Age}

Eleven studies assessed the association between age and mortality in elderly haemodialysis patients $[10,11,14$, $19,21,22,25,27,32,34,35]$. A random-effect model was used to analyse these eleven studies because there was heterogeneity between them $\left(p<0.001, \mathrm{I}^{2}=97 \%\right)$. The results showed that age was a risk factor for mortality in elderly haemodialysis population $(\mathrm{OR}=1.43,95 \%$ CI: 1.22-1.68), as shown in Fig. 2.

\section{Body mass index (BMI)}

Six studies assessed the association between body mass indexand mortality in elderly haemodialysis patients [11, $20,23,25,35,36]$. The results showed no heterogeneity between studies $\left(p=0.14, \mathrm{I}^{2}=40 \%\right)$, so we adopted a fixed-effect model to analyse these data. BMI $\geq 25$ was a protective factor for mortality in elderly haemodialysis population $(\mathrm{OR}=0.94,95 \% \mathrm{CI}$ : 0.92-0.96).

\section{Cardiovascular disease (CVD)}

Five studies appraised the association between CVD and mortality in elderly haemodialysis patients $[9,11,22,35$, 36]. Was used a random-effect model to analyse these 5 studies because of the heterogeneity between them ( $p=$ $\left.0.05, \mathrm{I}^{2}=58 \%\right)$. The results showed that CVD was not a

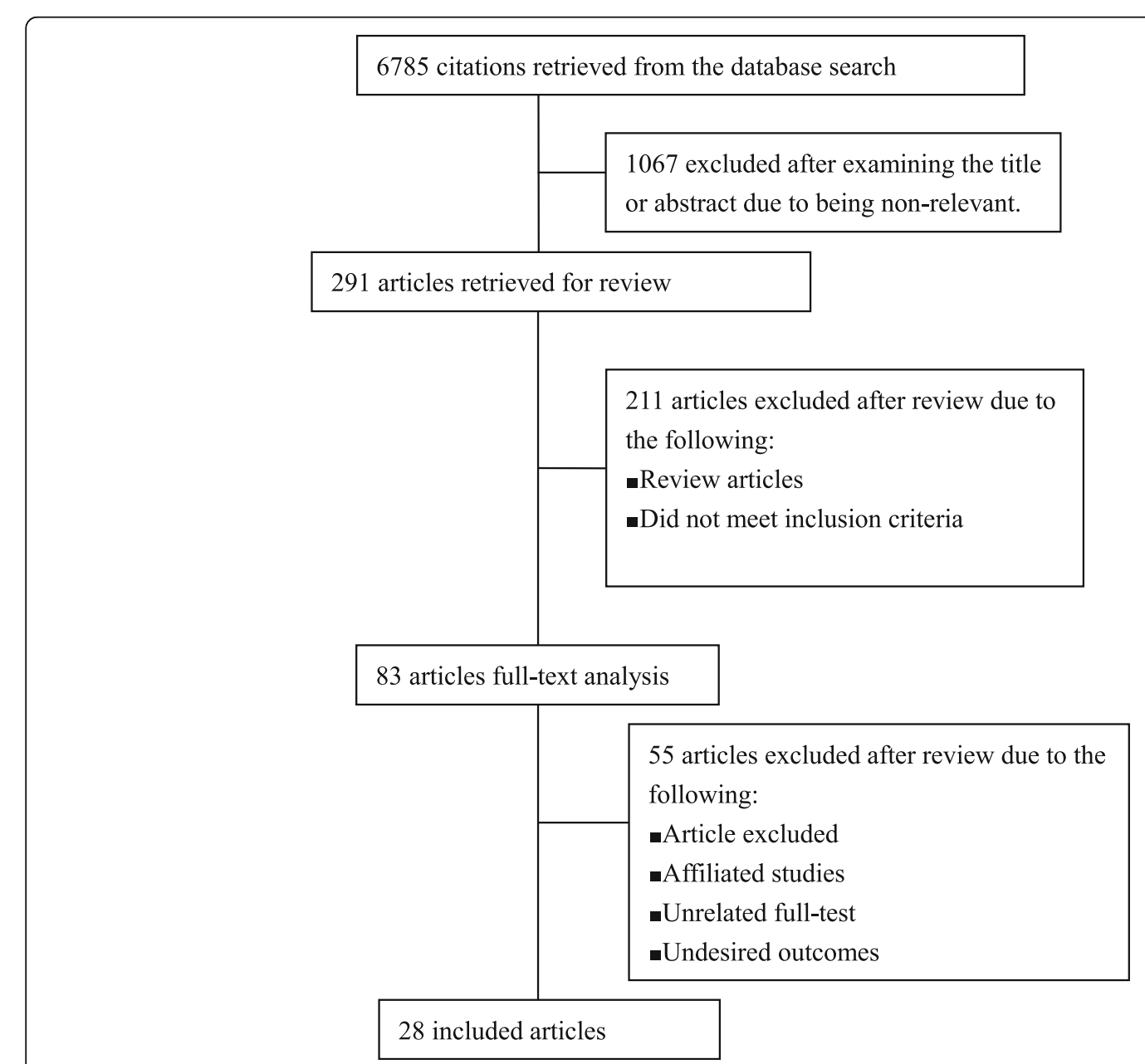

Fig. 1 Study selection process 


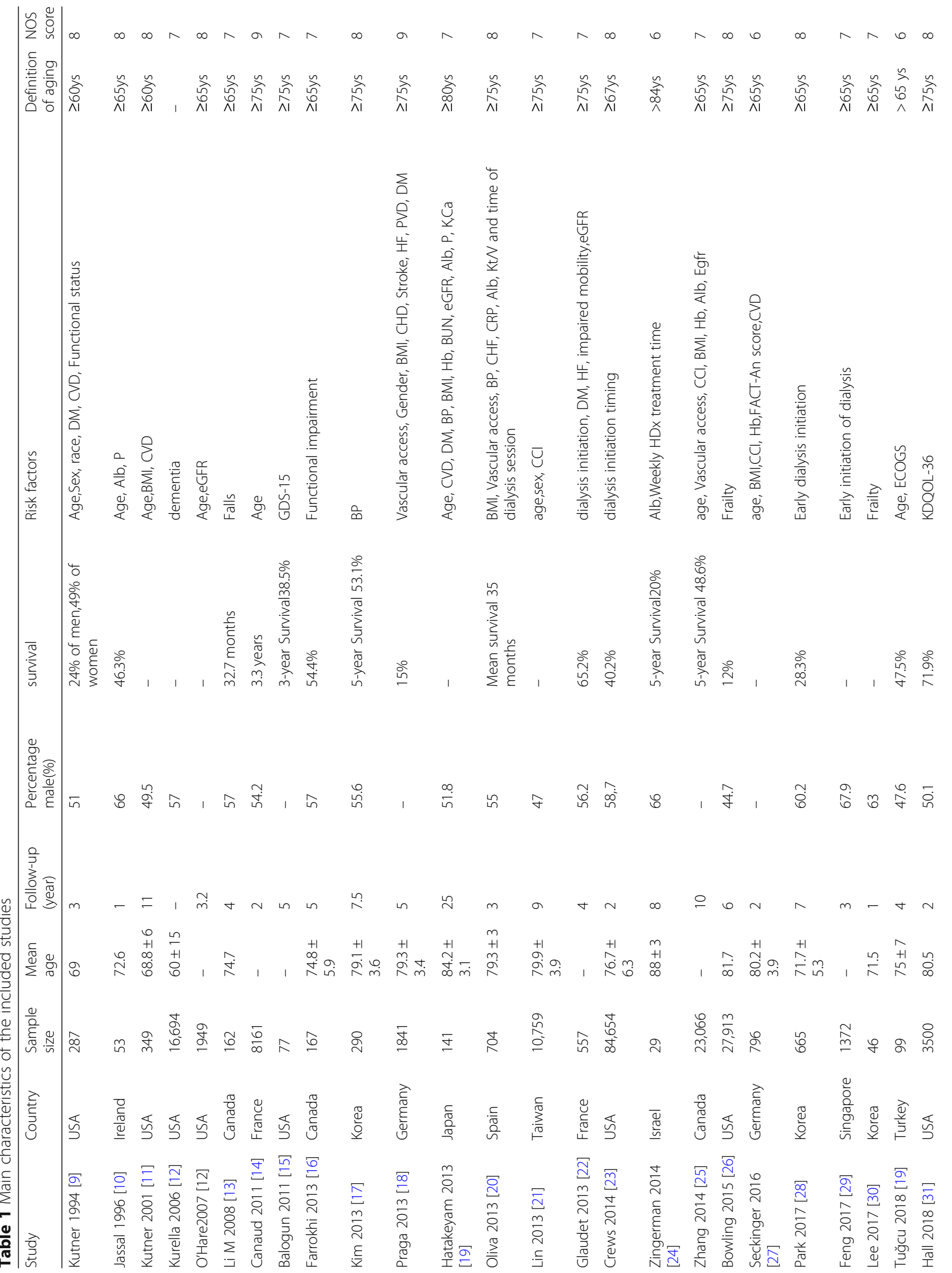




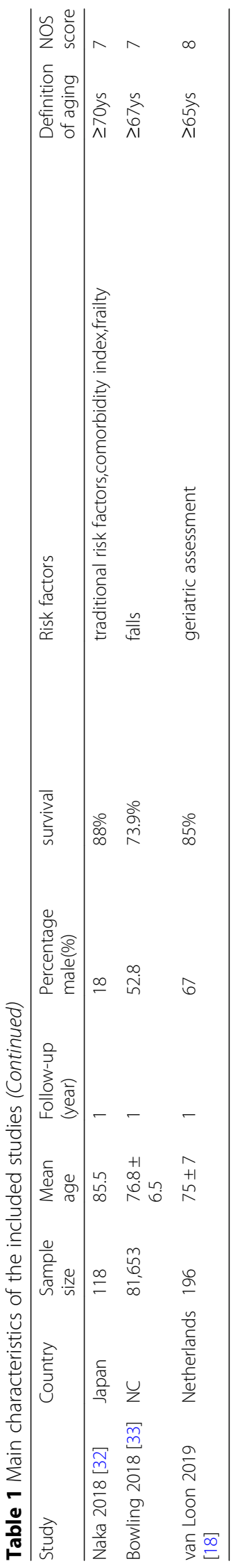




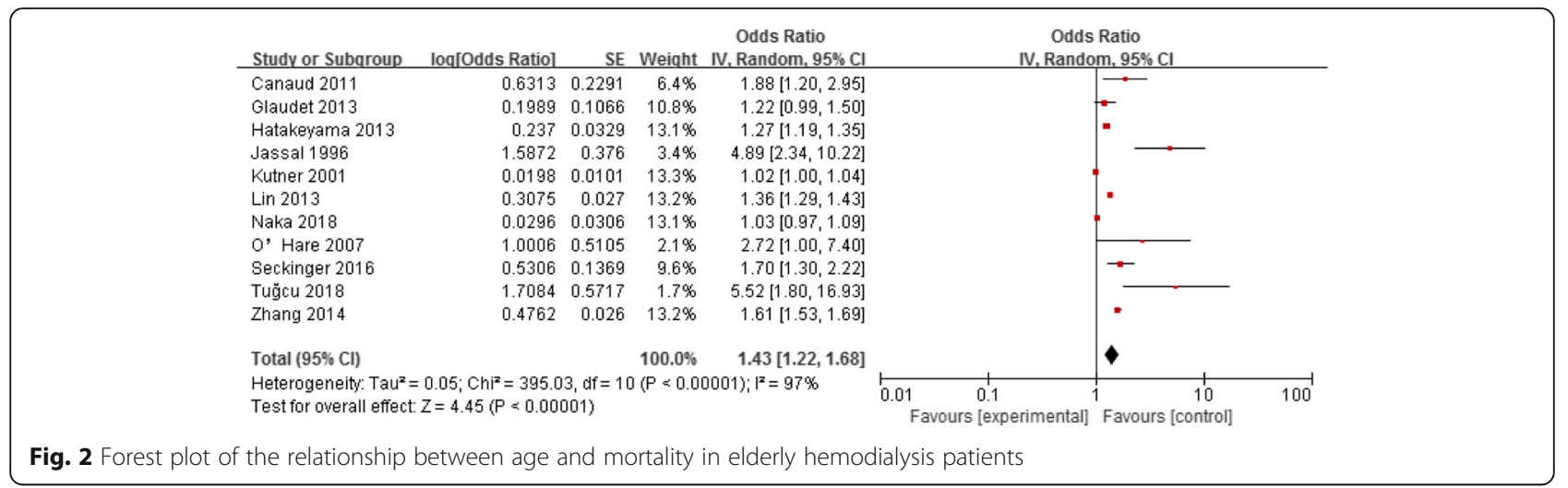

risk factor for mortality in elderly haemodialysis population $(\mathrm{OR}=1.20$, 95\% CI: $1.00-1.44)$.

\section{Diabetes mellitus (DM)}

We screened five studies assessing the association between DM and mortality in elderly haemodialysis patients $[9,11,22,35,36]$. The results showed no heterogeneity between studies $\left(p=0.72, \mathrm{I}^{2}=0 \%\right)$, so a fixed-effect model was used. Analysis of these 5 studies revealed that DM was a risk factor for mortality in aged haemodialysis population $(\mathrm{OR}=1.19,95 \% \mathrm{CI}$ : $1.06-$ 1.33).

\section{Central venous catheter dialysis}

Four studies assessed the association between central venous catheters and mortality in elderly haemodialysis patients $[20,22,25,36]$. The results showed heterogeneity between studies $\left(p=0.07, \mathrm{I}^{2}=58 \%\right)$, so the randomeffect model was used to analyse these data. Central venous catheter dialysis was a risk factor for mortality in aged haemodialysis population $(\mathrm{OR}=1.55,95 \% \mathrm{CI}$ : 1.38-1.75).

\section{Early-start dialysis}

Three studies assessed the association between dialysis initiation time and mortality in elderly haemodialysis patients $[19,23,28]$. The results showed no heterogeneity between studies $\left(p=0.26, \mathrm{I}^{2}=26 \%\right)$, so we used a fixedeffect model. Analysis of these 3 research studies showed that early dialysis was an infuencing factor for mortality in elderly haemodialysis population $(\mathrm{OR}=1.11,95 \% \mathrm{CI}$ : 1.08-1.14).

\section{Frailty}

Five studies assessed the association between frailty and mortality in elderly haemodialysis patients $[16,18,19$, $26,30]$. A fixed-effect model was used to analyse these five studies because there was no heterogeneity between them $\left(p<0.00001, \mathrm{I}^{2}=32 \%\right)$. The results showed that frailty was a risk factor for mortality in elderly haemodialysis population $(\mathrm{OR}=1.43,95 \% \mathrm{CI}: 1.31-1.56)$, as shown in Fig. 3.

\section{Functional impairment}

Seven studies assessed the association between functional impairment and mortality in elderly haemodialysis patients $[9,11,16,18,22,31,35]$. A random-effect model was used to analyse these data because there was heterogeneity between the studies ( $\left.p=0.0006, \mathrm{I}^{2}=75 \%\right)$. The results showed that functional impairment was a risk factor for mortality in elderly haemodialysis population $(\mathrm{OR}=1.45$, 95\% CI: 1.20-1.75), as shown in Fig. 4.

\section{Cognitive impairment}

Three studies assessed the association between cognitive impairment and mortality in elderly haemodialysis patients $[12,15,31]$. A fixed-effect model was used to analyse these data because there was no heterogeneity between studies $\left(p<0.00001, \mathrm{I}^{2}=0 \%\right)$. The results showed that cognitive impairment was a risk factor for

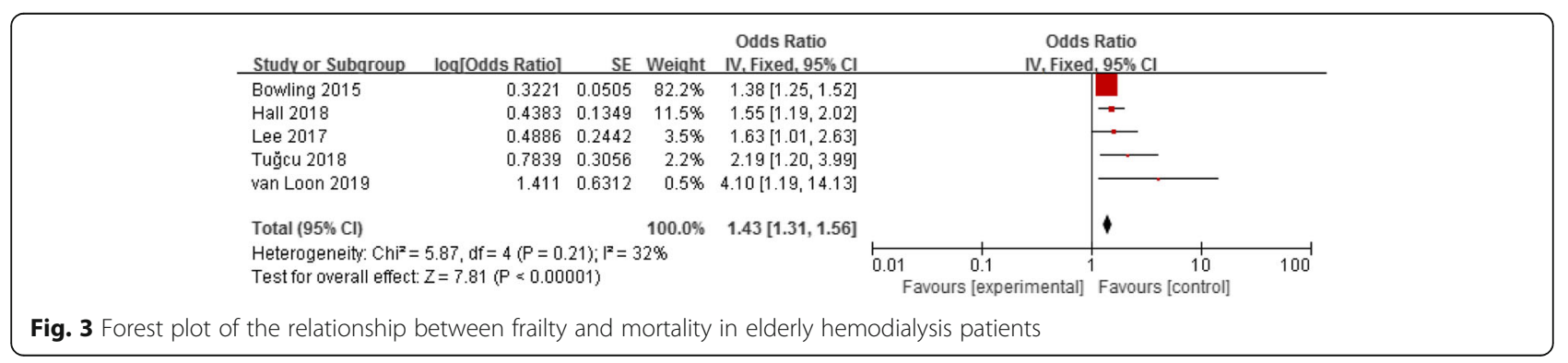




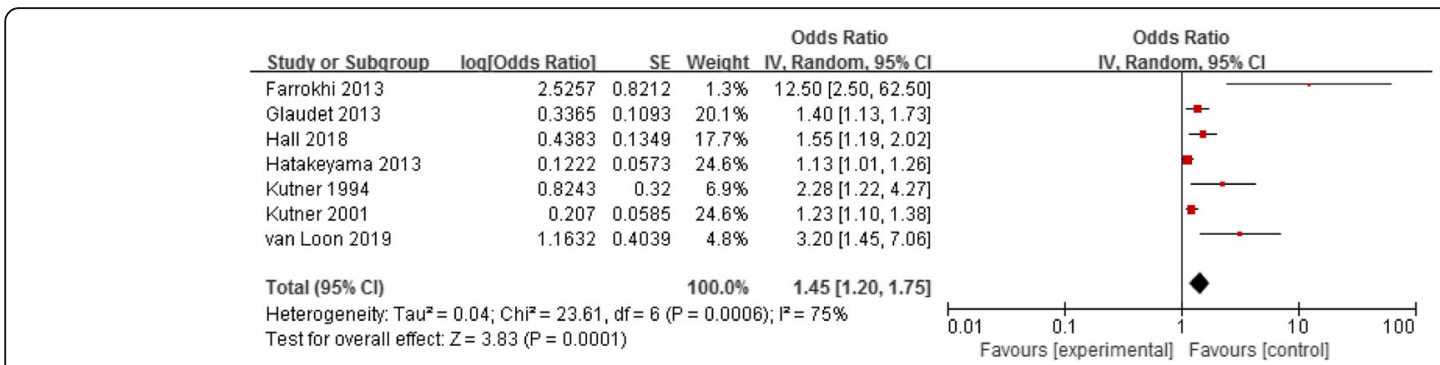

Fig. 4 Forest plot of the relationship between functional impairment and mortality in elderly hemodialysis patients

death in elderly haemodialysis population $(\mathrm{OR}=1.46$, 95\% CI: 1.32-1.62), as shown in Fig. 5.

\section{Falls}

Only two studies assessed the association between falls and mortality in elderly haemodialysis patients [13, 33]. The results showed that falls were a risk factor for death in elderly haemodialysis population $(\mathrm{OR}=1.14,95 \% \mathrm{CI}$ : 1.06-1.23).

\section{Sensitivity analysis and summary of the meta-analysis results of risk factors for mortality in elderly haemodialysis patients}

From the 28 selected studies [9-36], a summary of the meta-analysis results of risk factors for mortality in elderly haemodialysis patients was shown in Table 2 . The OR value and $95 \%$ CIs of each risk factor were assessed under the fixed-effect model and the random-effect model separately. The difference between the two results was small, indicating that the combined results had low sensitivity and stability.

\section{Publication bias}

The funnel plots expressed symmetric patterns for each outcome, as shown in Figs. 6 and 7. We conducted Begg's test to evaluate the publication bias using Stata software because the sample sizes of the outcomes included in this meta-analysis were small, which demonstrated no significant heterogeneity among the 28 studies.

\section{Discussion}

We evaluated 28 studies that composed of risk factors for mortality in elderly haemodialysis population in this study. This study supported the opinion that evaluation of geriatric senescence might promote to making decisions for dialysis by illustrating that multiple impaired factors are relevant to poor consequence [37-39].

Functional impairment is considered to be a contributor to subsequent disability, recurrent hospitalization, and decreased survival rate $[18,31,40]$. Loss of independent functioning has been recognized in geriatric dialysis patients [41-43]. Sensorial degeneration and sight defect are also not uncommon [44]. We found that functional impairment was a powerful, independently coherent predictor of mortality in elderly dialysis person. There is a requirement for early recognition of elderly haemodialysis patients who might get help from involvements in order to prevent or decrease geriatric impairment.

Cognitive impairment is not uncommon among dialysis patients [45]. This review showed that cognitive impairment in older haemodialysis patients is positively correlated to mortality. Older haemodialysis patients are also at potential risk of being befalled with Alzheimer's disease, and receiving this diagnose is associated with an increased mortality [46, 47]. Another study found that dementia was associated with an increased risk of death and dialysis drop out in adults aged over 75 years on dialysis [31]. Elderly dialysis patients should be considered to establish routine screening for cognitive impairment so as to recognize those at risk for related adverse consequences [12]. Large-scale studies to clear the vintage methods for detection, treatment and prevention of cognitive impairment are of critical necessity in this highrisk groups [48].

Other age-related comorbidities, such as falls, consult an independent and significant mortality risk for

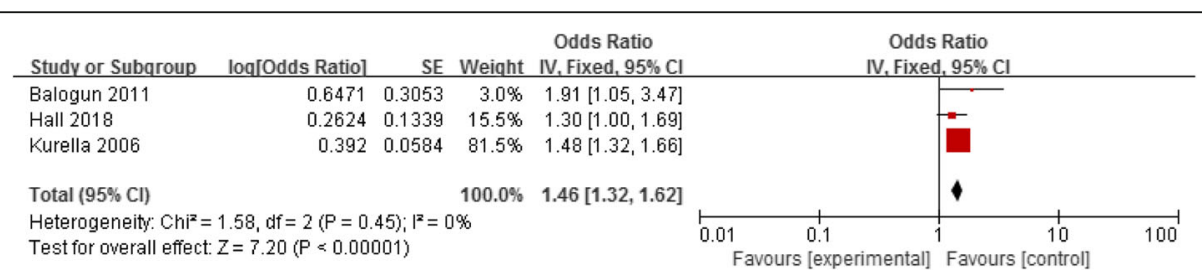

Fig. 5 Forest plot of the relationship between cognitive impairment and mortality in elderly hemodialysis patients 
Table 2 Comparison of meta-analysis results between fixed effect model and random effect model

\begin{tabular}{llc}
\hline Risk factors & Fixed effect model $[\mathrm{OR}(95 \% \mathrm{Cl})]$ & Random effect model $[\mathrm{OR}(95 \% \mathrm{Cl})]$ \\
\hline Age & $1.12(1.10-1.14)$ & $1.43(1.22-1.68)$ \\
CVD & $1.07(0.83-1.39)$ & $1.20(1.00-1.44)$ \\
DM & $1.19(1.06-1.33)$ & $1.19(1.06-1.33)$ \\
Vascular access CVC vs. AV & $1.53(1.44-1.62)$ & $1.55(1.38-1.75)$ \\
Early dialysis initiation & $1.11(1.08-1.14)$ & $1.18(1.01-1.37)$ \\
BMl>25 & $0.94(0.92-0.96)$ & $0.94(0.90-0.97)$ \\
Functional impairment & $1.21(1.12-1.31)$ & $1.55(1.16-2.07)$ \\
Cognitive impairment & $1.46(1.32-1.62)$ & $1.46(1.32-1.62)$ \\
Frailty & $1.43(1.31-1.56)$ & $1.53(1.29-1.83)$ \\
Falls & $1.14(1.06-1.23)$ & $1.14(1.06-1.23)$
\end{tabular}

geriatric dialysis population. Approximately $40 \%$ of elderly dialysis patients encounter one or more unexpected falls within one-year phase [13]. Multiple mediations have been performed to decrease fall rates and/or prevent damage associated with falls. These consist of multivariate evaluation and intervention, exercise moderating and the use of hip protectors in specific populations [13].

We also found that older age and more combined conditions (such as diabetes mellitus or hypertension) were correlated with higher mortality, which is well known in the general population. The observation that low BMI conferred a mortality risk paralleled the finding of frailty as a marker of early death. Increased mortality was also associated with early-start dialysis and with the use of a central venous catheter. The latter two points are well understood in the renal literature.

However, our meta-analysis had several limitations. First, the sample size of the included studies was too different and may have amplified the impact of individual studies on our results. Second, follow-up time affected the mortality of haemodialysis patients, which affected the accuracy of our meta-analysis. Third, this evidence is derived from a heterogeneous cohort of studies and the definitions of old age were different between our studies. Some of the research only included a small number of elderly patients aged over 80 years, which may have increased the mortality of haemodialysis patients and reduced the accuracy of our results. Additionally, the quality of this metaanalysis might be affected by the limitations at the review level (e.g., reporting bias) and at the outcome level (e.g., risk of bias).

\section{Conclusions}

This review described the impact of various characteristics on the risk of mortality in elderly patients undergoing haemodialysis. The mortality is high in

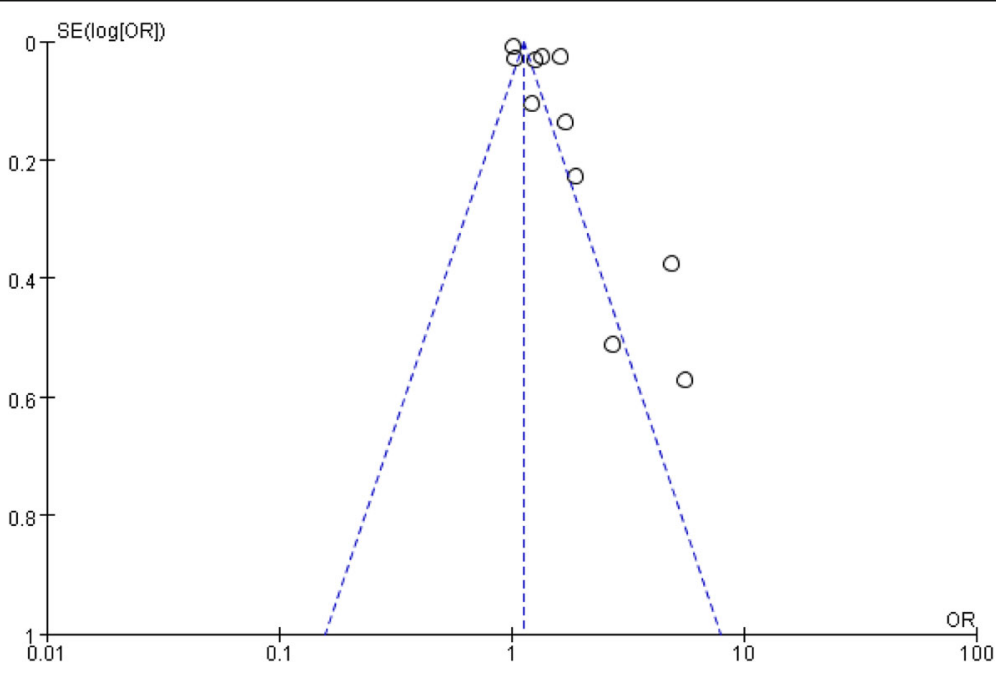

Fig. 6 Funnel plot of the relationship between age and mortality in elderly hemodialysis patients 


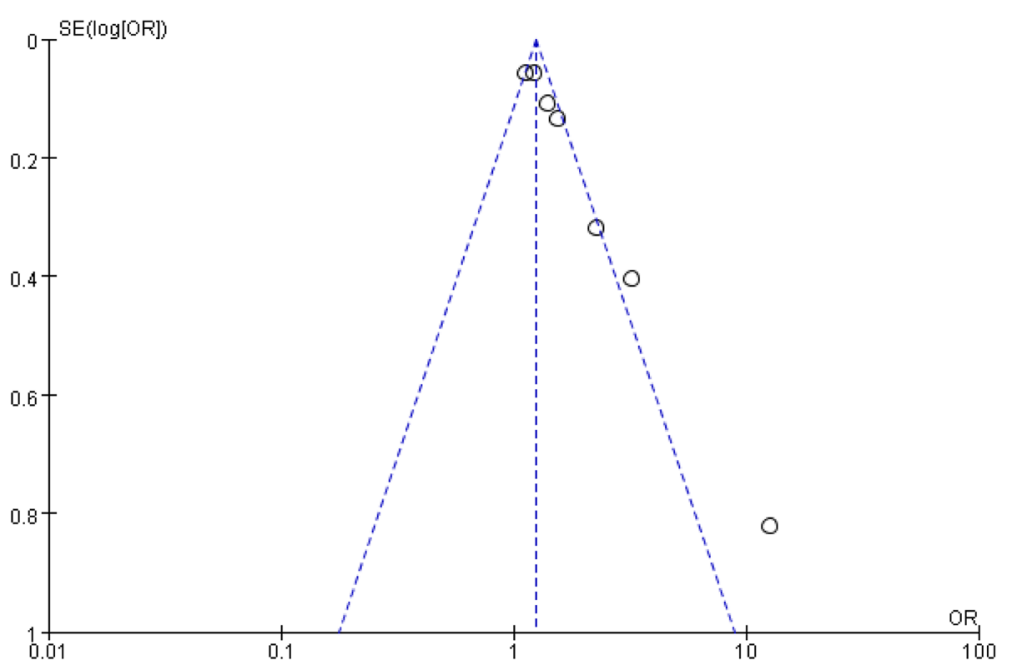

Fig. 7 Funnel plot of the relationship between functional impairment of dialysis mortality in elderly hemodialysis patients

geriatric haemodialysis patients who have functional and cognitive impairment and falls. Our findings may help determine the prognosis of geriatric dialysis patients. Large-scale studies are needed to address the changing world of nephrology and the challenges to nephrologists who are extremely interested in geriatric nephrology.

\section{Supplementary information}

Supplementary information accompanies this paper at https://doi.org/10. 1186/s12882-020-02026-X.

Additional file 1. PRISMA-P checklist.

Additional file 2. Example search strategy using PubMed.

\section{Abbreviations}

CKD: Chronic kidney disease; CVD: Cardiovascular disease; BMI: Body mass index; DM: Diabetes mellitus; OR: Odds ratio; HR: Hazard ratio; RR: Risk ratio; PRISMA: Preferred Reporting Items for Systematic reviews and Meta-Analyses

\section{Acknowledgements}

We thank all of the investigators who responded to our inquiries regarding their original studies.

\section{Authors' contributions}

Each author contributed to the design of the study and interpretation of the data. YHS, GYC, YFX, and XMC conceived and designed the study. YHS and GYC participated in the literature search, data analysis, and data interpretation. YHS drafted the manuscript. GYC and YFX revised the final manuscript. All authors read and approved the final manuscript.

\section{Funding}

This study was supported by the National Key Technology R\&D Program (2018YFA0108803, design of the study, collection and analysis of data) and the Science and Technology Project of Beijing (D181100000118004, interpretation of data and writing of the manuscript).

\section{Availability of data and materials}

All the data supporting the conclusions of this article are contained within the manuscript. The individual patient-level dataset was not made publicly available due to containing potentially identifying patient data; however, the study dataset may be made available from the authors upon request.
Ethics approval and consent to participate

This study protocol does not need ethics committee approval.

\section{Consent for publication}

Not applicable.

\section{Competing interests}

There are no conflicts of interest to declare. The coauthor Xiangmei Chen is the Editorial Board Member of BMC Nephrology.

Received: 9 November 2019 Accepted: 18 August 2020

Published online: 31 August 2020

\section{References}

1. Corbett RW, Brown EA. Conventional dialysis in the elderly: How lenient should our guidelines be? Semin Dial. 2018;31(6):607-11.

2. Chan KN, Chen Y, Lit Y, et al. A randomized controlled trial of exercise to prevent muscle mass and functional loss in elderlyhemodialysis patients: Rationale, study design, and baseline sample. Contemp Clin Trials Commun. 2019:19(15):100365.

3. Goto NA, Hamaker ME, Willems HC, et al. Accidental falling in communitydwelling elderly with chronic kidney disease. Int Urol Nephrol. 2019;51(1): $119-27$.

4. Plantinga LC, Patzer RE, Franch $H A$, et al. Serious fall injuries before and after initiation of hemodialysis among older ESRD patients in the United States: a retrospective cohort study. Am J Kidney Dis. 2017;70(1):76-83.

5. Iseri K, Carrero JJ, Evans M, et al. Major fractures after initiation of dialysis: incidence, predictors and association with mortality. Bone. 2020;17:115242 [Epub ahead of print].

6. Kooman JP, van der Sande FM, Leunissen KM. Prognosis in elderly patients with ESRD is quite variable and related to the presence of comorbidity and geriatric impairments. Kidney disease and aging: A reciprocal relation. Exp Gerontol. 2017:87(Pt B):156-9.

7. van Loon IN, Wouters TR, Boereboom FT, et al. The relevance of geriatric impairments in patients starting Dialysis: a systematic review. Clin J Am Soc Nephrol. 2016;11(7):1245-59.

8. Lo CK, Mertz D, Loeb M. Newcastle-Ottawa Scale: comparing reviewers' to authors' assessments. BMC Med Res Methodol. 2014:1(14):45.

9. Kutner NG, Lin LS, Fielding B, et al. Continued survival of older hemodialysis patients: investigation of psychosocial predictors. Am J Kidney Dis. 1994; 24(1):42-9.

10. Jassal SV, Douglas JF, Stout RW. Prognostic markers in older patients starting renal replacement therapy. Nephrol Dial Transplant. 1996;11(6):1052-7.

11. Kutner NG, Zhang R. Body mass index as a predictor of continued survival in older chronic dialysis patients. Int Urol Nephrol. 2001;32(3):441-8. 
12. Kurella M, Mapes DL, Port FK, et al. Correlates and outcomes of dementia among dialysis patients: the Dialysis Outcomes and Practice Patterns Study. Nephrol Dial Transplant. 2006;21(9):2543-8.

13. Li M, Tomlinson G, Naglie G, et al. Geriatric comorbidities, such as falls, confer an independent mortality risk to elderly dialysis patients. Nephrol Dial Transplant. 2008;23(4):1396-400.

14. Canaud B, Tong L, Tentori F, et al. Clinical practices and outcomes in elderly hemodialysis patients: results from the Dialysis outcomes and practice patterns study (DOPPS). Clin J Am Soc Nephrol. 2011;6(7):1651-62.

15. Balogun RA, Balogun SA, Kepple AL, et al. The 15-item geriatric depression scale as a predictor of mortality in older adults undergoing hemodialysis. J Am Geriatr Soc. 2011;59(8):1563-5.

16. Farrokhi F, Jassal SV. Routine use of an abbreviated 4-item scale to assess dependence in essential activities of dailyliving amongst elderly hemodialysis patients: a validation study. Int Urol Nephrol. 2013:45(1):259-64.

17. Kim HY, Kang YU, Kim CS, et al. Association of age and BP variability with long-term mortality in hemodialysis patients. Kidney Blood Press Res. 2013; 38(2-3):172-80.

18. van Loon IN, Goto NA, Boereboom FTJ, et al. Geriatric Assessment and the Relation with Mortality and Hospitalizations in Older Patients Starting Dialysis. Nephron. 2019;143(2):108-19.

19. Tuğcu M, Kasapoğlu U, Şahin G, et al. The Factors Affecting Survival in Geriatric Hemodialysis Patients. Int J Nephrol. 2018;10(2018):5769762.

20. Oliva JS, Roa LM, Lara A, et al. Survival and factors predicting mortality in hemodialysis patients over 75 years old. J Nephrol. 2013;26(1):129-35.

21. Lin YT, Wu PH, Kuo MC, et al. High cost and low survival rate in high comorbidity incident elderly hemodialysis patients. PLoS One. 2013;8(9): e75318.

22. Glaudet F, Hottelart C, Allard J, et al. The clinical status and survival in elderly dialysis: example of the oldest region of France. BMC Nephrol. 2013; 25(14):131.

23. Crews DC, Scialla JJ, Liu J, et al. Predialysis health, dialysis timing, and outcomes among older United States adults. J Am Soc Nephrol. 2014;25(2): 370-9.

24. Zingerman B, Korzets A, Ori Y, et al. The very old on hemodialysis: 8 years' experience in a single unit. Blood Purif. 2014;37(1):12-7.

25. Zhang JC, Al-Jaishi AA, Na Y, et al. Association between vascular access type and patient mortality among elderly patients on hemodialysis in Canada. Hemodial Int. 2014;18(3):616-24.

26. Bowling $\mathrm{CB}$, Zhang $\mathrm{R}$, Franch $\mathrm{H}$, et al. Underreporting of nursing home utilization on the CMS-2728 in older incident dialysis patients and implications for assessing mortality risk. BMC Nephrol. 2015;21(16):32.

27. Seckinger J, Dschietzig W, Leimenstoll G, et al. Morbidity, mortality and quality of life in the ageing haemodialysis population: results from the ELDE RLY study. Clin Kidney J. 2016;9(6):839-48.

28. Park JY, Yoo KD, Kim YC, et al. Early dialysis initiation does not improve clinical outcomes in elderly end-stage renal disease patients: a multicenter prospective cohort study. PLoS One. 2017;12(4):e0175830

29. Feng $L$, Jin $A Z$, Allen JC, et al. Timing of commencement of maintenance dialysis and mortality in young and older adults in Singapore. BMC Nephrol. 2017:18(1):176.

30. Lee SW, Lee A, Yu MY, et al. Is Frailty a Modifiable Risk Factor of Future Adverse Outcomes in Elderly Patients with IncidentEnd-Stage Renal Disease? J Korean Med Sci. 2017;32(11):1800-6.

31. Hall RK, Luciano A, Pieper C, et al. Association of Kidney Disease Quality of life (KDQOL-36) with mortality and hospitalization in older adults receiving hemodialysis. BMC Nephrol. 2018;19(1):11.

32. Naka T, Kohagura K, Kochi M, et al. Hyponatremia and mortality among very elderly residents in a geriatric health service facility. Clin Exp Nephrol. 2018; 22(6):1404-10

33. Bowling CB, Hall RK, Khakharia A, et al. Serious fall injury history and adverse health outcomes after initiating hemodialysis among older U.S. adults. J Gerontol A Biol Sci Med Sci. 2018;73(9):1216-21.

34. O'Hare AM, Choi Al, Bertenthal D, et al. Age affects outcomes in chronic kidney disease. J Am Soc Nephrol. 2007;18(10):2758-65.

35. Hatakeyama S, Murasawa H, Hamano I, et al. Prognosis of elderly Japanese patients aged $\geq 80$ years undergoing hemodialysis. Sci World J. 2013;9(2013): 693514.

36. Praga M, Merello Jl, Palomares I, et al. Type of vascular access and survival among very elderly hemodialysis patients. Nephron Clin Pract. 2013;124(12):47-53.
37. Bowling CB, Plantinga L, Hall RK, et al. Association of Nondisease-Specific Problems with mortality, long-term care, and functional impairment among older adults who require skilled nursing care after Dialysis initiation. Clin J Am Soc Nephrol. 2016;11(12):2218-24.

38. Jassal SV, Chiu E, Hladunewich M. Loss of independence in patients starting dialysis at 80 years of age or older. N Engl J Med. 2009;361(16):1612-3.

39. Kurella Tamura M, Covinsky KE, Chertow GM, et al. Functional status of elderly adults before and after initiation of dialysis. N Engl J Med. 2009; 361(16):1539-47.

40. Wick JP, Turin TC, Faris PD, et al. A clinical risk prediction tool for 6-month mortality after dialysis initiation among older adults. Am J Kidney Dis. 2017; 69(5):568-75.

41. Bossola M, Di Stasio E, Antocicco M, et al. Functional impairment is associated with an increased risk of mortality in patients on chronic hemodialysis. BMC Nephrol. 2016;7(1):72.

42. Jassal SV, Karaboyas A, Comment LA, et al. Functional Dependence and Mortality in the International Dialysis Outcomes and PracticePatterns Study (DOPPS). Am J Kidney Dis. 2016;67(2):283-92.

43. Wongrakpanich $S$, Susantitaphong $P$, Isaranuwatchai $S$, et al. Dialysis Therapy and Conservative Management of Advanced Chronic Kidney Disease in the Elderly: A Systematic Review. Nephron. 2017;137(3):178-89.

44. Chiu E, Markowitz SN, Cook WL, et al. Visual impairment in elderly patients receiving long-term hemodialysis. Am J Kidney Dis. 2008;52(6):1131-8.

45. Shea YF, Lee MC, Mok MM, et al. Prevalence of cognitive impairment among peritoneal dialysis patients: a systematic review and meta-analysis. Clin Exp Nephrol. 2019;23(10):1221-34.

46. Yiannopoulou KG, Anastasiou Al, Kyrozis A, et al. Donepezil treatment for Alzheimer's disease in chronic Dialysis patients. Case Rep Nephrol Dial. 2019; 9(3):126-36

47. McAdams-DeMarco MA, Daubresse M, Bae S, et al. Dementia, Alzheimer's disease, and mortality after hemodialysis initiation. Clin J Am Soc Nephrol. 2018;13(9):1339-47.

48. Tsuruya $\mathrm{K}$, Yoshida $\mathrm{H}$. Brain atrophy and cognitive impairment in chronic kidney disease. Contrib Nephrol. 2018;196:27-36.

\section{Publisher's Note}

Springer Nature remains neutral with regard to jurisdictional claims in published maps and institutional affiliations.

Ready to submit your research? Choose BMC and benefit from

- fast, convenient online submission

- thorough peer review by experienced researchers in your field

- rapid publication on acceptance

- support for research data, including large and complex data types

- gold Open Access which fosters wider collaboration and increased citations

- maximum visibility for your research: over $100 \mathrm{M}$ website views per year

At $\mathrm{BMC}$, research is always in progress.

Learn more biomedcentral.com/submission 